\title{
Parâmetros clínicos e bioquímicos de pacientes renais crônicos em hemodiálise com níveis elevados de fosfatase alcalina
}

\author{
Clinical and biochemical parameters of chronic renal patients in hemodialysis with high alkaline \\ phosphatase levels
}

\section{DOI: $10.37111 /$ braspenj.2019344005}

Maria Rita Fonseca Dias'

Raissa Castro Ribeiro'

Vanessa Fernandes Lobão

Michel Garcia Maciel2

Andréa Dias Reis ${ }^{3}$

Luciana Pereira Pinto Dias ${ }^{4}$

Karina Silva Cordeiro ${ }^{5}$

Mylenne Cardim Ferreira ${ }^{1}$

Isabelle Christine Vieira da Silva Martins ${ }^{6}$

\section{Unitermos:}

Fosfatase alcalina. Calcificação fisiológica. Insuficiência renal crônica. Diálise renal. Doenças cardiovasculares.

\section{Keywords:}

Alkaline phosphatase. Calcification, physiologic. Renal insufficiency, chronic. Renal dialysis. Cardiovascular diseases.

\section{Endereço para correspondência:}

Michel Garcia Maciel

SQN 405, bloco I, apto 308 - Brasília, DF, Brasil - CEP

70846-090

E-mail: mgmaciel@gmail.com

\section{Submissão}

14 de agosto de 2019

Aceito para publicação

27 de outubro de 2019

\begin{abstract}
RESUMO
Introdução: A doença renal crônica (DRC) é um problema de saúde pública global, que implica em má condição de saúde e elevado risco de doenças cardiovasculares, contribuindo com altas taxas de mortalidade, a qual tem sido associada a altos níveis de fosfatase alcalina (FA) neste público. O objetivo deste estudo foi comparar os parâmetros clínicos e bioquímicos em relação aos níveis de fosfatase alcalina de pacientes renais crônicos em hemodiálise. Método: Estudo transversal e analítico, realizado em 93 pacientes de ambos os sexos, com idade igual ou superior a 18 anos, de um centro de hemodiálise na cidade de Belém, Pará. A coleta de dados incluiu entrevista por meio de questionário semiestruturado e consulta aos prontuários. A estatística descritiva foi composta por média, desvio padrão, mediana, intervalo interquartil, frequência relativa e absoluta. Para análise da fosfatase alcalina, utilizou-se o intervalo interquartil com valor de 120 U/L. Resultados: Do total de 93 pacientes, 58 eram homens, com idade média de 54,5 \pm 4,5 anos. A amostra foi dividida em dois grupos: o primeiro, com fosfatase alcalina baixa - menor que $120 \mathrm{U} / \mathrm{L}(\mathrm{n}=50 ; 82,24 \pm 21,67)$; o segundo, com fosfatase alcalina alta - maior ou igual a $120 \mathrm{U} / \mathrm{L}(\mathrm{n}=43 ; 288,82 \pm 300,05)$. Após análise do perfil clínico e bioquímico de cada grupo, encontrou-se diferença estatística nos valores de paratormônio e hemoglobina, em que o grupo com fosfatase alcalina elevada obteve maiores níveis de paratormônio e valores mais baixos de hemoglobina. Observou-se considerável prevalência de fosfatase elevada em pacientes renais crônicos em hemodiálise. Conclusão: $O$ estudo identificou paratormônio alto e hemoglobina mais baixa nos pacientes com fosfatase alcalina elevada. Ressalta-se a importância de monitoração e correção dos níveis de FA, quando possível.
\end{abstract}

\section{ABSTRACT}

Introduction: Chronic kidney disease (CKD) is a global public health problem, which implies poor health and high risk of cardiovascular disease contributing to high mortality rates, which has been associated with high levels of alkaline phosphatase (AF) in this public. The aim of this study was to compare clinical and biochemical parameters in relation to alkaline phosphatase levels of chronic renal patients on hemodialysis. Methods: Cross-sectional and analytical study, performed in 93 male and female patients, aged 18 years or older, from a hemodialysis center in the city of Belém, Pará. Data collection included interviews through a semi-structured questionnaire and consultation of medical records. Descriptive statistics consisted of mean, standard deviation, median, interquartile range, relative and absolute frequency. For alkaline phosphatase analysis, the interquartile range of $120 \mathrm{U} / \mathrm{L}$ was used. Results: Of the 93 patients, 58 were men with a mean age of $54.5 \pm 4.5$ years. The sample was divided into two groups: the first, with low alkaline phosphatase - less than $120 \mathrm{U} / \mathrm{L}(n=50 ; 82.24 \pm 21.67)$; and the second, with high alkaline phosphatase - greater than or equal to $120 \mathrm{U} / \mathrm{L}(\mathrm{n}=43 ; 288.82 \pm 300.05)$. After analyzing the clinical and biochemical profile of each group, we found a statistical difference in the values of parathyroid hormone and hemoglobin, in which the group with high alkaline phosphatase obtained higher parathyroid hormone levels and lower hemoglobin values. Considerable prevalence of elevated phosphatase was observed in chronic renal patients on hemodialysis. Conclusion: The study identified high parathyroid hormone and lower hemoglobin in patients with high alkaline phosphatase. We emphasize the importance of monitoring and correcting AF levels, when possible.

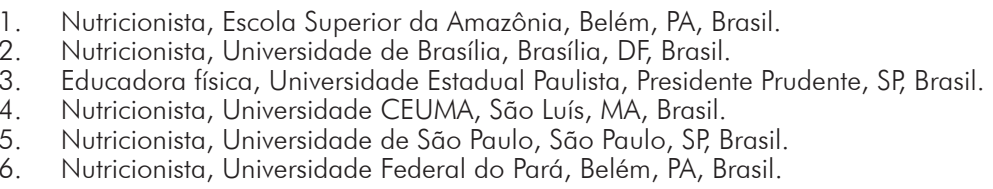




\section{INTRODUÇ̃̃O}

A doença renal crônica (DRC) é um problema de saúde pública global, que implica em má condição de saúde e alto risco de doenças cardiovasculares, contribuindo com altas taxas de mortalidade'. Algumas particularidades da insuficiência renal podem estar envolvidas nessas elevadas taxas, como calcificação aórtica, maiores níveis de creatinina e anemia frequente ${ }^{2}$.

A elevada mortalidade entre renais crônicos tem sido associada a altos níveis de fosfatase alcalina $(F A)^{3}$. Essa enzima encontra-se no sangue, assim como na superfície de células hepáticas, dos ductos biliares e do tecido ósseo, por isso é um marcador importante de desordens nessas estruturas $^{4}$. Na DRC, distúrbios enzimáticos, como da FA, parecem causar aumento nos níveis de fosfato inorgânico $\left(P_{\mathrm{i}}\right)$, o qual tem sido associado ao processo de calcificação vascular ${ }^{5}$.

A maior retenção de fósforo $(P)$ também está envolvida na fisiopatologia do distúrbio mineral ósseo (DMO), comum nos estágios mais avançados da DRC. Caracteriza-se por alterações ósseas progressivas, em que, além do papel do P, a deficiência de cálcio, por conta do déficit de calcitriol, também é um ponto-chave do distúrbio. Dessa forma, seu monitoramento deve ser realizado principalmente pela dosagem trimestral do paratormônio (PTH), assim como a FA necessita ser avaliada em conjunto, por ser um marcador de remodelação óssea ${ }^{6}$.

O Kidney Disease Improving Global Outcomes (KDIGO) recomenda, a partir do estágio 3 da insuficiência renal, a monitoração dos níveis séricos de FA periodicamente, e com maior frequência, se houver aumento do PTH. No entanto, não estipula um ponto de corte ou um intervalo adequado para manutenção dos níveis séricos da FA .

Apesar disso, estudos com populações de pacientes renais crônicos, que avaliaram diferentes níveis de FA com prevalência de doenças ósseas e mortalidade, sugerem que valores de até $120 \mathrm{U} / \mathrm{L}$ poderiam ser considerados seguros, sendo menos associados a estas comorbidades ${ }^{8-10}$.

Portanto, o objetivo deste estudo foi comparar os parâmetros clínicos e bioquímicos em relação aos níveis de fosfatase alcalina de pacientes renais crônicos em hemodiálise.

\section{MÉTODO}

Tratou-se de um estudo do tipo transversal e analítico, no qual foram avaliados 93 pacientes de um Centro de Hemodiálise na cidade de Belém, Pará. Incluíram-se na pesquisa pacientes de ambos os sexos, com idade igual ou superior a 18 anos, em condições plenas de comunicação, com tempo mínimo de hemodiálise igual ou superior a seis meses, sem histórico de realização de transplante renal prévio e que concordaram e assinaram o Termo de Consentimento Livre e Esclarecido (TCLE). Foram excluídos pacientes com problemas mentais, portadores de hepatite $\mathrm{C}$ e outras doenças infecciosas.

Elaborou-se o trabalho conforme as diretrizes e normas regulamentadas de pesquisa envolvendo seres humanos, sendo aprovado pelo Comitê de Ética em Pesquisa do Núcleo de Medicina Tropical da Universidade Federal do Pará, sob protocolo n 1.505.937/16.

A coleta de dados foi realizada por meio de entrevista aos pacientes, utilizando-se um questionário semiestruturado com dados clínicos, além de consulta aos prontuários, para obtenção de dados laboratoriais e clínicos.

\section{Variáveis}

Utilizou-se a ureia sérica pré e pós-dialítica para o cálculo do Kt/Vsp. A adequação da diálise foi avaliada por meio do $\mathrm{Kt} / \mathrm{V}$ single pool, segundo a fórmula de Daugirdas ${ }^{11}$.

As medidas antropométricas foram obtidas após sessões de hemodiálise, e realizadas pelo próprio serviço de nutrição da clínica, sob acompanhamento dos pesquisadores. Aferiuse o peso pós-dialítico em balança eletrônica, e a altura por meio de estadiômetro. $O$ índice de massa corporal (IMC) foi calculado pela razão do peso corporal e quadrado da altura, sendo classificado, para adultos, segundo a Organização Mundial da Saúde ${ }^{12}$, para idosos, utilizou-se a classificação específica para este grupo ${ }^{13}$.

Verificou-se a análise do risco cardiovascular pela equação pré-estabelecida da razão triglicerídeo (TG)/ lipoproteína de alta densidade (HDL-c), obtida a partir de valores de TG e HDL-c plasmáticos, considerando-se como risco cardiovascular valores maiores que 2,5 para mulheres e maiores que 3,5 para homens ${ }^{14}$.

A comparação dos grupos (<120 U/L e $\geq 120 \mathrm{U} / \mathrm{L}$ ) para FA foi obtida pela divisão interquartil da amostra total, na qual se definiu 120 como o ponto de corte amostral. Representou-se a estatística descritiva por média, desvio padrão, mediana, intervalo interquartil, frequência relativa e absoluta. Aplicou-se o teste Kolmogorov-Smirnov para verificar a normalidade das variáveis.

Utilizou-se o teste de Levini nas variáveis independentes e com distribuição normal: creatinina, hemoglobina e CaxP, seguido da aplicação do teste T de Student não-pareado. Nas variáveis independentes e não-paramétricas, foi utilizado o teste Mann Whitney. Para variáveis independentes e dicotômicas, usou-se o teste de Qui Quadrado. Naquelas com frequência absoluta menor que 5 foi aplicado o Teste Exato de Fisher. Realizou-se a estatística pelo software SPSS versão 21.0 , considerando-se $\alpha=5 \%$, ou seja, significância com o $\mathrm{p}<0,05$. 


\section{RESULTADOS}

A amostra foi composta por 93 pacientes, sendo a maioria $(62,36 \%)$ do sexo masculino. Os pacientes foram alocados em dois grupos: o primeiro com FA menor que 120 $\mathrm{U} / \mathrm{L}(\mathrm{n}=50 ; 82,24 \pm 21,67)$, e o segundo com FA maior ou igual a $120 \mathrm{U} / \mathrm{L}(\mathrm{n}=43 ; 288,82 \pm 300,05)$.

Quanto à presença de diabetes melitus (DM), a maioria dos pacientes não apresentou a patologia em ambos os grupos: 56\% no grupo com FA menor que $120 \mathrm{U} / \mathrm{L}$, e $55,8 \%$ no grupo com FA maior ou igual a $120 \mathrm{U} / \mathrm{L}$, não havendo diferença estatística $(p=0,986)$. $O$ tempo de HD também foi analisado, e independentemente dos níveis de FA, a maior parte dos pacientes possuía entre 1 e 5 anos de $\mathrm{HD}$, sendo $70 \%$ no grupo com FA menor que $120 \mathrm{U} / \mathrm{L}$ e $72,1 \%$ no grupo com FA maior ou igual a $120 \mathrm{U} / \mathrm{L}$, sem diferenças estatísticas.

Os parâmetros clínicos e bioquímicos foram comparados entre os grupos, e não houve homogeneidade apenas para as variáveis ferritina $(p=0,037)$, paratormônio $(p=0,004)$ e hemoglobina $(p=0,035)$ (Tabela 1).

Quando realizada a análise do perfil de cada grupo, observou-se significância estatística nos valores de PTH $(p=0,001)$ e Hb $(p=0,014)$ (Tabela 2).

\section{DISCUSSÃO}

O estudo demonstrou que, aproximadamente, metade da frequência de pacientes em terapia dialítica apresentou níveis elevados de FA. Observou-se diferença entre os pacientes com FA alta e baixa, em relação aos níveis de paratormônio e hemoglobina. O achado é preocupante, uma vez que a FA elevada é fator de risco independente para fraturas ósseas.

Em um caso-controle com 354 renais crônicos, esses mesmos pacientes apresentaram mais eventos cardiovasculares e mortalidade ${ }^{15}$. O maior risco de mortalidade parece estar relacionado à doença cardiovascular, por consequência da calcificação aórtica ${ }^{16}$.

Ao analisar o quadro clínico de pacientes renais, dois estudos distintos no Paraná, um com 132 indivíduos e outro com 26, encontraram valores medianos de FA de $129 \mathrm{U} / \mathrm{L}$ e de $202,7 \mathrm{U} / \mathrm{L}$, respectivamente, sendo semelhantes ao obtido no presente estudo ${ }^{17,18}$.

Além disso, observou-se uma relação existente entre maiores valores de fosfatase alcalina e o hiperparatireoidismo (HPTS). O HPTS é considerado uma das causas de mortalidade em pacientes com DRC por causar fraturas, deformidades ósseas e decréscimo do tempo de vida ${ }^{17,19}$.

Tabela 1 - Parâmetros clínicos e bioquímicos conforme os diferentes níveis de fosfatase alcalina de pacientes em hemodiálise de Belém, Pará.

\begin{tabular}{|c|c|c|c|}
\hline & $\begin{array}{c}\text { Fosfatase Alcalina } \\
\begin{array}{c}<120 \mathrm{U} / \mathrm{L} \\
\mathrm{n}=50\end{array}\end{array}$ & $\begin{array}{c}\text { Fosfatase Alcalina } \\
\begin{array}{c}\geq 120 \mathrm{U} / \mathrm{L} \\
\mathrm{n}=43\end{array}\end{array}$ & $p$ valor \\
\hline \multicolumn{4}{|l|}{ Clínico } \\
\hline Idade & $59(41-64)$ & $50(38-60)$ & 0,101 \\
\hline Ktv & $1,29(1,15-1,40)$ & $1,33(1,18-1,43)$ & 0,277 \\
\hline Albumina (g/dL) & $4,20(3,80-4,50)$ & $4,20(3,90-4,40)$ & 0,685 \\
\hline Ferritina (ng/mL) & $340(139,25-487,50)$ & $451(294-813)$ & $0,037^{*}$ \\
\hline Fósforo (mg/dL) & $5(4,18-5,73)$ & $4,50(3,90-5,50)$ & 0,176 \\
\hline Cálcio (mg/dL) & $8,72(8,32-9,06)$ & $8,96(8,48-9,28)$ & 0,105 \\
\hline $\mathrm{TG}(\mathrm{mg} / \mathrm{dL})$ & $124(98,25-257,25)$ & $159(107-230)$ & 0,874 \\
\hline TG/HDL & $3,07(2-5,89)$ & $3,56(2,17-6,31)$ & 0,890 \\
\hline Creatinina $(\mathrm{mg} / \mathrm{dL})^{\mathrm{a}}$ & $7,37 \pm 2,28$ & $7,90 \pm 2,38$ & 0,354 \\
\hline Hemoglobina $(\mathrm{mg} / \mathrm{dL})^{\mathrm{a}}$ & $10,87 \pm 2,23$ & $9,95 \pm 1,96$ & $0,035^{\star}$ \\
\hline CaxPa & $43,31 \pm 12,29$ & $41,76 \pm 13,60$ & 0,548 \\
\hline
\end{tabular}


Tabela 2-Classificação dos parâmetros clínicos e bioquímicos conforme os diferentes níveis de fosfatase alcalina de pacientes em hemodiálise de Belém, Pará.

\begin{tabular}{|c|c|c|c|}
\hline & $\begin{array}{c}\text { Fosfatase Alcalina } \\
<120 \mathrm{U} / \mathrm{L} \\
\mathrm{n}=50\end{array}$ & $\begin{array}{c}\text { Fosfatase Alcalina } \\
\geq 120 \mathrm{U} / \mathrm{L} \\
n=43\end{array}$ & $\mathrm{p}$ valor \\
\hline \multicolumn{4}{|l|}{ Clínico } \\
\hline $\mathrm{Kt} / \mathrm{V}$ & & & 0,825 \\
\hline$<1,2$ & $15(30 \%)$ & $12(27,9 \%)$ & \\
\hline Magreza/eutrofia & 37 (74\%) & $27(62,8 \%)$ & \\
\hline Excesso de peso & $13(26 \%)$ & $16(37,2 \%)$ & \\
\hline \multicolumn{4}{|l|}{ Bioquímico } \\
\hline Albumina $(\mathrm{g} / \mathrm{dL})^{\mathrm{a}}$ & & & 1,000 \\
\hline Ferritina (ng/mL) & & & 0,097 \\
\hline$<100$ & $11(22 \%)$ & $4(9,3 \%)$ & \\
\hline$>100$ & $39(78 \%)$ & $39(90,7 \%)$ & \\
\hline Fósforo (mg/dL) & & & 0,378 \\
\hline$<5,5$ & $33(66 \%)$ & $32(74,4 \%)$ & \\
\hline$\geq 5,5$ & 17 (34\%) & $11(25,6 \%)$ & \\
\hline Cálcio (mg/dL) & & & 0,405 \\
\hline$<8,5$ & $18(36 \%)$ & $12(27,9 \%)$ & \\
\hline$<190$ & $40(80 \%)$ & $39(90,7 \%)$ & \\
\hline$\geq 190$ & $10(20 \%)$ & $4(9,3 \%)$ & \\
\hline Razão TG/HDL & & & 0,412 \\
\hline Sem risco cardiovascular & $24(48 \%)$ & $17(39,5 \%)$ & \\
\hline Com risco cardiovascular & $26(52 \%)$ & $26(60,5 \%)$ & \\
\hline Creatinina (mg/dL) & & & $b$ \\
\hline$<1,3$ & - & - & \\
\hline$\geq 1,3$ & $50(53,8 \%)$ & $43(46,2 \%)$ & \\
\hline Hemoglobina (g/dL) & & & $0,014^{*}$ \\
\hline$<12$ & $35(70 \%)$ & $39(90,7 \%)$ & \\
\hline$\geq 12$ & $15(30 \%)$ & $4(9,3 \%)$ & \\
\hline CaxP (mg/dL) & & & 0,484 \\
\hline$<55$ & $43(86 \%)$ & $39(90,7 \%)$ & \\
\hline$\geq 55$ & $7(14 \%)$ & $4(9,3 \%)$ & \\
\hline
\end{tabular}

a: Exato de Fisher; *: $p<0,05$.

Ktv = depuração da ureia do dialisador, multiplicada pelo tempo de tratamento e dividido pelo volume de distribuição de ureia (medida de adequação de hemodiálise); IMC = Índice de massa corporal; HDL = Lipoproteína de alta densidade; TG = Triglicerídeos; CaxP = Relação do produto cálcio $x$ fósforo séricos. 
Desordens no metabolismo ósseo pela disfunção renal, como o HPTS, parecem ser os mesmos mecanismos que levaram à FA aumentada nos pacientes deste estudo.

No Brasil, em 52 pacientes foram identificadas alterações ecocardiográficas, algumas das quais correlacionadas a níveis circulantes de $\mathrm{PTH}^{20}$. Outro resultado semelhante, com 230 nigerianos, identificou a FA elevada mais frequente nos pacientes com HPTS, associados à hiperfosfatemia e à hipocalcemia ${ }^{21}$.

Um estudo avaliou pacientes renais em uso ou não de medicamentos como quelantes de $\mathrm{P}$ e calcimiméticos, sendo observada melhora dos níveis de FA de 248,8 $\mathrm{U} / \mathrm{L}$ para 121,4 , naqueles aderentes ao tratamento medicamentoso 22 .

estudo verificou que, nos pacientes com FA > $120 \mathrm{U} / \mathrm{L}$, os níveis de hemoglobina apresentaram diferença, quando comparados aos pacientes com FA < $120 \mathrm{U} / \mathrm{L}$. Nesse sentido, há várias causas de anemia renal, como diminuição do hormônio eritropoietina (EPO), redução da sobrevivência dos glóbulos vermelhos e fibrose da medula óssea. Esta última pode ocorrer pelo HTPS, pois o excesso de secreção de PTH leva à fibrose do tecido medular, interferindo na eritropoiese. $E$, proporcionalmente à extensão da fibrose na medula óssea, aumenta-se a dose de EPO para atingir uma resposta adequada do hematócrito ${ }^{23}$.

Um estudo multicêntrico, com 53 adultos na Nova Zelândia, demonstrou que a FA sérica também esteve associada à resistência ao tratamento com agentes estimulantes de eritropoiese (AEE), a qual se manteve persistente, mesmo após a correção para outras causas de anemia. Contudo, os mecanismos dessa possível relação ainda são desconhecidos ${ }^{24}$.

A pesquisa, de fácil reprodução e baixo custo, sugeriu um perfil com características que impactam diretamente na qualidade de vida dos pacientes analisados, mesmo considerando-se que a amostra foi do tipo não-probabilística, e de não ter sido aplicado o método padrão para avaliação da mineralização óssea.

\section{CONCLUSÃO}

O grupo com níveis elevados de FA demonstrou baixos níveis de hemoglobina e altos níveis de paratormônio, os quais são parâmetros usuais de prática clínica, que podem auxiliar na tomada de decisão. Diante dos resultados, ressalta-se a importância da monitoração e correção dos níveis de FA nos pacientes renais em hemodiálise.

\section{REFERÊNCIAS}

1. Di Angelantonio E, Chowdhury R, Sarwar N, Aspelund T, Danesh J, Gudnason V. Chronic kidney disease and risk of major cardiovascular disease and non-vascular mortality: prospective population based cohort study. BMJ. 2010;341:c4986.

2. Martin LC, Franco RJS. A doença renal como fator de risco cardiovascular. Arq Bras Cardiol. 2005;85(6):432-6.

3. Rhee CM, Molnar MZ, Lau WL, Ravel V, Kovesdy CP, Mehrotra R, et al. Comparative mortality-predictability using alkaline phosphatase and parathyroid hormone in patients on peritoneal dialysis and hemodialysis. Perit Dial Int. 2014;34(7):732-48.

4. Schoppet M, Shanahan CM. Role for alkaline phosphatase as an inducer of vascular calcification in renal failure? Kidney Int. 2008;73(9):989-91.

5. Moe SM, Chen NX. Pathophysiology of vascular calcification in chronic kidney disease. Circ Res. 2004;95(6):560-7.

6. Custódio MR, Canziani MEF, Moysés RMA, Barreto FC, Neves CL, Oliveira RB, et al. Protocolo clínico e diretrizes terapêuticas para o tratamento do hiperparatireoidismo secundário em pacientes com doença renal crônica. Bras J Nefrol. 2013;35(4):308-22.

7. Kidney Disease: Improving Global Outcomes (KDIGO) CKD-MBD Update Work Group. KDIGO 2017 Clinical practice guideline update for the diagnosis, evaluation, prevention, and treatment of chronic kidney disease-mineral and bone disorder (CKD-MBD). Kidney Int Suppl. 2017;7(1):1-59.

8. Park JC, Kovesdy CP, Duong U, Streja E, Rambod M, Nissenson AR, et al. Association of serum alkaline phosphatase and bone mineral density in maintenance hemodialysis patients. Hemodial Int. 2010;14(2):182-92.

9. Li J, Molnar MZ, Zaritsky JJ, Sim JJ, Streja E, Kovesdy CP, et al. Correlates of parathyroid hormone concentration in hemodialysis patients. Nephrol Dial Transplant. 2013;28(6):1516-25.

10. Pan J, Hu H, Zhang W, Chen J, Dou X. Value of serum alkaline phosphatase for predicting 2-year fracture in patients with chronic kidney disease on dialysis. Nan Fang Yi Ke Da Xue Xue Bao. 2018;38(9):1095-9.

11. Daugirdas JT. Second generation logarithmic estimates of singlepool variable volume kt/V: an analysis of error. J Am Soc Nephrol. 1993;4(5):1205-13.

12. World Health Organization (WHO). WHO Technical Report Series, 854: Physical status: the use and interpretation of anthropometry. Geneva: World Health Organization; 1995.

13. Lipschitz DA. Screening for nutritional status in the elderly. Prim Care. 1994;21(1):55-67.

14. Salazar MR, Carbajal HA, Espeche GT, Sisnieguez CEL, Balbín E, Dulbecco CA, et al. Relation among the plasma triglyceride/ high-density lipoprotein cholesterol concentration ratio, insulin resistance, and associated cardio-metabolic risk factors in men and women. Am J Cardiol. 2012;109(12):1749-53.

15. Li C, Chen XM, Li Y, Zhou YL, Yan JN, Du XG. Factors and outcome of renal osteodystrophy-associated initial fragility fracture in end-stage renal disease patients. Kidney Dis (Basel). 2019;5(2):118-25.

16. Peyro-Shabani A, Nabahati M, Saber-Sadeghdoust MA, Soleymani MJ, Oliaei F. Risk factors associated with aortic calcification in hemodialysis patients. Caspian J Intern Med. 2018;9(4):347-52.

17. Jaqueto M, Delfino VDA, Bortolasci CC, Barbosa DS. Morimoto HK, Frange RFN, et al. Os níveis de PTH estão relacionados com estresse oxidativo e inflamação em pacientes renais crônicos em hemodiálise? J Bras Nefrol. 2016;38(3):288-95.

18. Bucharles SGE, Barreto FC, Riella MC. Impacto de cinacalcete em marcadores do metabolismo mineral de pacientes em diálise portadores de hiperparatireoidismo secundário grave. Braz J Nephrol. 2019;41(3):336-44. 
19. Loncar G, Bozic B, Cvetinovic N, Dungen HD, Lainscak M, von Haehling S, et al. Secondary hyperparathyroidism prevalence and prognostic role in elderly males with heart failure. J Endocrinol Invest. 2017;40(3):297-304.

20. Costa AFP, Diniz Junior PRT, Santos VM. Achados ecocardiográficos em pacientes com hiperparatireoidismo secundário. Int $\mathrm{J}$ Cardiovasc Sci. 2016;29(1):13-8.

21. Gimba ZM, Abene EE, Agbaji OOO, Agaba EI. Secondary hyperparathyroidism among Nigerians with chronic kidney disease. Afri Health Sci. 2018;18(2):446-57.
22. Santos JB, Almeida PHRF, Lemos LB, Lemos GS. Evidências clínicas da adesão medicamentosa de pacientes dialíticos crônicos. Rev Enferm UFPE on line. 2018;12(12):3254-62.

23. Tanaka M, Komaba H, Fukagawa M. Emerging association between parathyroid hormone and anemia in hemodialysis patients. Ther Apher Dial. 2018;22(3):242-5.

24. Badve SV, Zhang L, Coombes JS, Pascoe EM, Cass A, Clarke P, et al. Association between serum alkaline phosphatase and primary resistance to erythropoiesis stimulating agents in chronic kidney disease: a secondary analysis of the HERO trial. Can J Kidney Health Dis. 2015;2:33.

Local de realização do estudo: Centro de Hemodiálise Monteiro Leite, Belém, PA, Brasil.

Conflito de interesse: Os autores declaram não haver.

Foi apresentado como trabalho de destaque no XXIII Congresso Brasileiro de Nutrição Parenteral e Enteral, entre 20 a 23 de outubro de 2019, em Foz do Iguaçu-PR. 